\title{
Global representation in psychiatric research
}

\author{
Michael Zhang ${ }^{1}$ BS, Dawson W. Hedges ${ }^{2}$ MD and Bruce L. Brown ${ }^{3}$ PhD
}

${ }^{1}$ Graduate student, Department of Psychology, Brigham Young University, Provo, Utah, USA email michael_zhang@byu.edu

2Professor, Department of Psychology, and Neuroscience Center, Brigham Young University, Provo, Utah, USA

2Professor of Psychology, Brigham Young University, Provo, Utah, USA

The authors acknowledge Jenny Lake, Brian Larson, Helena

Mavromatis and Jamie Moses for their help in data collection.
To monitor global representation in the psychiatric literature, we compared publication rates in the ten psychiatric journals with the highest impact factors in 1998 and 2008 by world regions. In both 1998 and 2008, North America, Northern Europe, Western Europe and Oceania produced the majority of psychiatric research papers published in these journals, despite representing only a small fraction of the world's population. This suggests that much of the world's population continues to be underrepresented in highly influential psychiatric journals.

Psychiatric research productivity differs substantially between countries and world regions as measured by publication in journals with a high impact factor. For instance, comparatively little research published in such psychiatric journals comes from low- and middle-income nations (Patel \& Kim, 2007; Mari et al, 2010). While the disparity between high- and low-income nations in producing and publishing in peer-reviewed psychiatric journals is well described (Patel \& Kim, 2007; Mari et al, 2010) and understandable given the economic constraints inherent to lower-income countries, little research has investigated how well the nations producing considerable amounts of influential psychiatric research represent the world's population.

In 2001, Patel \& Sumathipala compared numbers of publications in six high-impact psychiatric journals over 3 years $(1996-98)$ by 'Euro-American' countries (including countries in Western Europe, North America and Oceania) and the rest of the world and found considerable underrepresentation by countries not from Euro-America in the influential psychiatric literature - only $6 \%$ of the research was published by countries that together accounted for over $90 \%$ of the world's population. They and other authors have offered prescriptions for increasing highimpact publication among international psychiatric researchers (Patel \& Sumathipala, 2001; Maj, 2005; Coverdale et al, 2007; Balon et al, 2008).

In this study, we extend the work of Patel \& Sumathipala (2001) by examining whether recommendations to facilitate more influential research and publications from underrepresented world regions have been successful and by estimating how well research published in psychiatric journals with a high impact factor represents the world's population. To do so, we replicate parts of Patel
\& Sumathipala's study but use additional journals in 1998 and compare the results with findings a decade later, in 2008. While much of the previous research on this topic has focused on publication disparities between high-income countries and low- and middle-income countries, our primary objective is to examine how psychiatric research reported in these journals represents the world's population. Furthermore, we briefly outline some future directions for research to monitor global representation in the psychiatric literature.

\section{Methods}

From the Journal Citations Report Science Edition for 2008, we extracted the impact factor for each of the listed psychiatric journals. Impact factors can be considered an estimate of the influence a journal has on research. We selected the ten psychiatric journals with the highest 1 -year and 5 -year impact factors in 2008.

From the published author affiliation section of all papers published in the ten journals with the highest 1-year and 5-year impact factors in 2008, and in these same journals in 1998 (which were also among the journals with the highest impact factors at that time), we extracted the country of the institutional affiliation of the first author of each paper and tallied the number of first authors by country. We combined the published articles by country into world regions as defined by the Population Reference Bureau, a widely accepted source for regional populations.

We divided the total number of first authors for each world region by the population of the region according to population estimates obtained from the Population Reference Bureau to obtain an estimate of the ratio of articles published to population in each world region during 1998 and 2008. Finally, we compared the number of articles published (again, as a population ratio) for each region in 1998 and 2008 to examine whether underrepresented regions in 1998 had become better represented a decade later.

\section{Results}

The ten journals in psychiatry with the highest 1-year and 5-year impact factors in 2008 were: Archives of General Psychiatry, Molecular Psychiatry, American Journal of Psychiatry, Biological Psychiatry, Neuropsychopharmacology, Schizophrenia Bulletin, British Journal of Psychiatry, Journal of Clinical Psychiatry, Journal of Child Psychology and Psychiatry and Journal of the American Academy of Child and Adolescent Psychiatry. The top ten journals were the 
Table 1

Number of papers published in the top ten psychiatric journals (by impact factor) from each of the 18 Population Reference Bureau regions in 1998 and 2008, number per million persons in each of those years, and change in number per million 1998-2008

\begin{tabular}{|c|c|c|c|c|c|}
\hline \multirow{2}{*}{ Region } & \multicolumn{2}{|c|}{$\begin{array}{l}\text { Number of papers } \\
\text { published }\end{array}$} & \multicolumn{2}{|c|}{$\begin{array}{l}\text { Papers/million } \\
\text { persons }\end{array}$} & \multirow{2}{*}{$\begin{array}{l}\text { Change in number } \\
\text { of papers/ } \\
\text { million persons, } \\
\text { 1998-2008 }\end{array}$} \\
\hline & 1998 & 2008 & 1998 & 2008 & \\
\hline Northern Africa & 0 & 0 & 0.00 & 0.00 & 0.00 \\
\hline Western Africa & 0 & 0 & 0.00 & 0.00 & 0.00 \\
\hline Eastern Africa & 0 & 0 & 0.00 & 0.00 & 0.00 \\
\hline Middle Africa & 0 & 0 & 0.00 & 0.00 & 0.00 \\
\hline Southern Africa & 1 & 2 & 0.02 & 0.03 & 0.01 \\
\hline North America & 685 & 899 & 2.28 & 2.64 & 0.36 \\
\hline Central America & 0 & 1 & 0.00 & 0.01 & 0.01 \\
\hline Caribbean & 0 & 0 & 0.00 & 0.00 & 0.00 \\
\hline South America & 3 & 12 & 0.01 & 0.03 & 0.02 \\
\hline Western Asia & 19 & 23 & 0.11 & 0.10 & -0.01 \\
\hline South Central Asia & 4 & 5 & 0.00 & 0.00 & 0.00 \\
\hline South-East Asia & 0 & 0 & 0.00 & 0.00 & 0.00 \\
\hline East Asia & 20 & 53 & 0.01 & 0.03 & 0.02 \\
\hline Northern Europe & 165 & 225 & 1.75 & 2.29 & 0.54 \\
\hline Western Europe & 75 & 180 & 0.41 & 0.95 & 0.54 \\
\hline Eastern Europe & 0 & 4 & 0.00 & 0.01 & 0.01 \\
\hline Southern Europe & 31 & 57 & 0.22 & 0.37 & 0.15 \\
\hline Oceania & 26 & 66 & 0.91 & 1.90 & 0.99 \\
\hline Total & 1029 & 1527 & & & \\
\hline
\end{tabular}

same for both 1-year and 5-year impact factors, although the order was not identical.

In 1998, among the 18 world regions as classified by the Population Reference Bureau, North America, Northern Europe, Western Europe and Oceania had the highest paper/population ratios (Table 1), producing 951 of the 1029 articles (92.4\%) published in the ten journals in 1998 while representing only $10.3 \%$ of the world's population.

The results were similar in 2008 - the North American, Northern European, Oceanic and Western European regions had the highest rates of publication (Table 1), producing $89.7 \%$ of the 1527 papers published in these journals despite containing as a group only $9.8 \%$ of the world's population. Further, North America, Northern Europe, Oceania and Western Europe showed the greatest increases between 1998 and 2008 in publications per head of population (Table 1, Fig. 1).

\section{Discussion}

We found that the vast majority of psychiatric research published in journals with a high impact factor in 2008 came from lead authors located in world regions that represented only a small part of the worlds' population, a publication

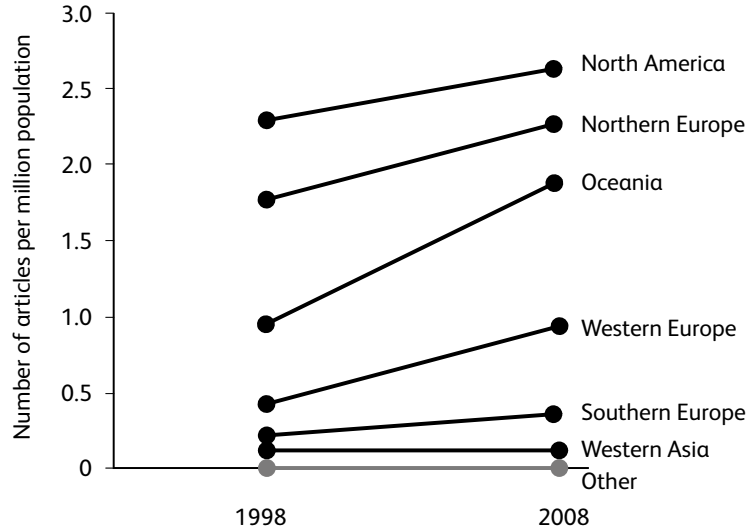

Fig. 1

Comparison of the numbers of articles published per million population in 1998 and 2008, for the 18 Population Reference Bureau world regions. The grey line at the bottom of the graph represents the remaining 12 unlabelled regions (Northern Africa, Western Africa, Eastern Africa, Middle Africa, Southern Africa, Central America, Caribbean, South America, South Central Asia, South-East Asia, East Asia, Eastern Europe), superimposed on each other

pattern essentially unchanged from 1998 (Patel \& Sumathipala, 2001). North America, Northern Europe, Oceania and Western Europe continued to produce most of the papers published in the top ten psychiatric journals, and few articles published in these journals came from lead authors in Africa, Asia or South America, regions that accounted for the majority of the world's population.

In fact, publication rates in these journals increased from 1998 to 2008 in North America, Northern Europe, Oceania and Western Europe (Table 1, Fig. 1). Although many regions showed little or no increase in publication per million people, some with low publication rates in 1998, such as Southern Africa, South America and East Asia, had small increases in publication rates between 1998 and 2008, with, for example, South America increasing from 0.01 to 0.03 and East Asia increasing from 0.01 to 0.03 . While encouraging, these increases were smaller in absolute terms than the increases seen in North America, Northern Europe, Oceania and Western Europe, with, for example, Oceania increasing from 0.91 to 1.90 and Western Europe increasing from 0.41 to 0.95 , suggesting that the factors underlying the disparities in publication rates in 1998 remained largely intact in 2008.

These findings suggest that psychiatric research published in journals with a high impact factor may represent only a small part of the world's population, despite strategies recommended by researchers and institutions to address the imbalance (Patel \& Sumathipala, 2001; Maj, 2005; Coverdale et al, 2007; Balon et al, 2008) and the expansion of global mental health initiatives in the same period (Balon et al, 2008).

We recognise that selecting those journals with a high impact factor is only one way to identify influential research. Future research could 
examine collaborations between authors in different world regions (Maj, 2005) or the actual subject pools used in studies (Patel \& Kim, 2007). Acceptance rates for submissions from underrepresented regions to the journals could also be compared with acceptance rates for submissions from overrepresented regions (Patel \& Kim, 2007). We also realise that psychiatric researchers from underrepresented world regions could be improving their publication rates in psychiatric journals with lower impact factors, and this is another possibility to explore in future research.

Despite previous recommendations to improve global representation in psychiatric research, much of the world's population still appears to be underrepresented in highly influential psychiatric journals. More effective actions need to be taken to achieve truly globally representative psychiatric research (Maj, 2005).

\section{References}

Balon, R., Roberts, L. W., Coverdale, J., et al (2008) Globalization of medical and psychiatric education and the focus of Academic Psychiatry on the success of 'international' authors. Academic Psychiatry, 32, 151-153.

Coverdale, J., Roberts, L. W., Louie, A. K., et al (2007) Enhancing the international status of Academic Psychiatry. Academic Psychiatry, 31, 177-179

Maj, M. (2005) Psychiatric research in low- and middle-income countries: the need for concrete action. Acta Psychiatrica Scandinavica, 111, 329-330.

Mari, J. J., Patel, V., Kieling, C., et al (2010) The 5/95 gap in the indexation of psychiatric journals of low- and middle-income countries. Acta Psychiatrica Scandinavica, 121, 152-156.

Patel, V. \& Kim, Y.-R. (2007) Contribution of low- and middleincome countries to research published in leading general psychiatry journals, 2002-2004. British Journal of Psychiatry, 190, 77-78.

Patel, V. \& Sumathipala, A. (2001) International representation in psychiatric literature: survey of six leading journals. British Journal of Psychiatry, 178, 406-409.
RESEARCH PAPER

\section{The diagnostic validity of depression scales and clinical judgement in the Kurdistan region of Iraq}

\author{
Zerak Al-salihy ${ }^{1}$ MBCHB MRCPsych, Twana A. Rahim² MBCHB FIBMSPsych, \\ Mahmud Q. Mahmud, ${ }^{3}$ Asma S. Muhyaldin ${ }^{4}$ and Alex J. Mitchell ${ }^{5}$ MBBS MRCPsych
}

${ }^{1}$ Specialty Registrar Level 5 (ST5) in Old Age Psychiatry, Norfolk and Suffolk Foundation Trust (NSFT), Bury St Edmunds, Suffolk, UK, email zeraksalihy@yahoo. com

${ }^{2}$ Senior Lecturer in Psychiatry and Behavioural Sciences, Faculty of Medicine, Hawler Medical University, Erbil, Iraq

${ }^{3}$ General Psychiatrist, Azadi Medical Centre, Erbil, Iraq 4Psychiatry Registrar, Tairawa Health Centre, Erbil, Iraq

${ }^{5}$ Consultant and Honorary Senior Lecturer, University of Leicester,
We aimed to find the depression rating scale with the greatest accuracy when applied by psychiatrists in Iraqi Kurdistan. We recruited 200 patients with primary depression and 200 controls living in the Kurdistan region of Iraq. The Mini International Neuropsychiatry Inventory (MINI) was used as a gold standard for DSM-IV depression. We also used: the two-item and the nine-item versions of the Patient Health Questionnaire (PHQ2, PHQ9), the Hospital Anxiety and Depression Scale (HADS), the Calgary Depression Scale for Schizophrenia (CDSS) and the Centre for Epidemiological Studies Depression (CES-D) scale. Interviews were performed by psychiatrists who also rated their clinical judgement using the Clinical Global Impression (CGI) scale and other mental health practitioners. All scales and tools performed with high accuracy and reliability. The least accurate tool was the PHQ2; however, with only two items it was efficient. Sensitivity and specificity for all tools were above $90 \%$. Clinicians using the CGI were accurate in their clinical judgement. The CDSS appeared to be the most accurate scale for DSM-IV major depression and the PHQ2 the most efficient. However, only the CDSS appeared to offer an advantage over psychiatrists' judgement.
Üstün et al (2004) estimated that depression is the fourth leading cause of global disease burden. The burden of depression on the healthcare system is equally significant, with an estimated US national annual medical cost of approximately $\$ 26$ billion in 1990 (Broadhead et al, 1990; Greenberg et al, 1993). The National Comorbidity Survey Replication (NCS-R), conducted with people aged at least 18 years, found a 12 -month prevalence of $9.5 \%$ for any DSM-IV mood disorder, with $6.7 \%$ for major depression and $1.5 \%$ for dysthymia (Kessler et al, 2005). A mental health survey in Iraq which was conducted in collaboration with World Health Organization in 2007 showed that 'anxiety' was the most common class of disorders (13.8\%) and major depressive disorder (MDD) the most common disorder (7.2\%) (Alhasnawi et al, 2009; World Health Organization, 2009)

The extensive literature on screening and case-finding for depression has been reviewed elsewhere. Screening for depression has been supported by recommendations from the US Preventive Services Task Force (Agency for Healthcare Research and Quality, 2002), the UK National Institute for Health and Clinical Excellence (2004) (NICE) and the Canadian Task Force on Preventive Health Care (MacMillan et al, 2005). Our aim was to find the tool with the highest accuracy relative to a robust gold standard. 